\title{
Aberrant Intranuclear Localization of Biotin, Biotin- binding Enzymes, and $\beta$-Catenin in Pregnancy-Related Endometrium and Morule-Associated Neoplastic Lesions
}

Ayako Gamachi, M.D., Kenji Kashima, M.D., Ph.D., Tsutomu Daa, M.D, Ph.D., Yukio Nakatani, M.D., Ph.D., Masahiko Tsujimoto, M.D., Ph.D., Shigeo Yokoyama, M.D., Ph.D. Department of Pathology, Oita Medical University (AG, KK, TD, SY), Oita; Department of Pathology, Yokohama City University Medical Center (YN), Yokohama; and Department of Pathology, Osaka Police Hospital (MT), Osaka, Japan

Biotin-rich intranuclear inclusions, also known as optically clear nuclei, have been observed in various neoplastic and non-neoplastic lesions. They look like nuclei of herpesvirus-infected cells and cause a false-positive immunohistochemical result by avidin-biotin-complex (ABC) method. In the literature, all types of neoplastic lesions with intranuclear inclusions, with one exception, have been characteristically associated with squamoid structures known as morules. By contrast, all reported nonneoplastic lesions with such inclusions lacked morules and were confined to pregnancy-related endometrium. In the present study, adding unreported types of morule-associated neoplastic lesions, we investigated the distribution of biotin, biotin-binding enzymes, and $\beta$-catenin in these lesions by immunohistochemical staining. We detected the intranuclear localization of biotin and of two mitochondrial biotin-binding enzymes (pyruvic acid carboxylase and propionyl CoA carboxylase) in all lesions examined, regardless of whether they were neoplastic or non-neoplastic and irrespective of the presence or absence of morules. The intranuclear localization of $\beta$-catenin was detected in all neoplastic lesions with morules and in ovarian endometrioid adenocarcinoma without morules, but not in non-neoplastic endometrial lesions. These results suggest the following conclusions: (1) lesions with biotin-rich intranuclear inclusions can be classified as non-neoplastic/pregnancy-related endometrial and as neoplastic/pregnancy-unrelated or

Copyright $\odot 2003$ by The United States and Canadian Academy of Pathology, Inc.

VOL. 16, NO. 11, P. 1124, 2003 Printed in the U.S.A.

Date of acceptance: July 14, 2003.

Address reprint requests to: Shigeo Yokoyama, M.D., Ph.D., Department of Pathology, Oita Medical University, Hasama-machi, Oita 879-5593, Japan; fax: +81-97-586-5686; e-mail: yokoyama@oita-med.ac.jp.

DOI: 10.1097/01.MP.0000092953.20717.48 morular category; (2) the intranuclear biotin in both types of lesion is found in conjunction with biotin-binding enzymes. However, the role of $\beta$-catenin in morule-associated neoplastic lesions, the relationship between $\beta$-catenin and biotin/ biotin-binding enzymes, and the mechanism of migration of biotin and biotin-binding enzymes from the cytoplasm to the nucleus remain unclear.

KEY WORDS: $\beta$-Catenin, Biotin, Biotin-binding enzymes, Intranuclear inclusion, Morule.

Mod Pathol 2003;16(11):1124-1131

Biotin is a form of vitamin B that acts as a coenzyme in reactions catalyzed by biotin-binding enzymes that include pyruvic acid carboxylase (PAC) and propionyl CoA carboxylase (PCC), which are involved in gluconeogenesis; acetyl CoA carboxylase (ACC), which is involved in fatty acid biosynthesis; carbamoyl phosphate synthetase I (CPS), which is involved in the urea cycle; carbamoyl phosphate synthetase II, which is involved in pyrimidine synthesis; and methyl-crotonyl carboxylase (MCC), which is involved in leucine metabolism. Among these biotin-binding enzymes, PAC, PCC, CPS-I, and MCC are localized in mitochondria, whereas ACC and carbamoyl phosphate synthetase II are found in the cytosol. Large amounts of biotin are present in the liver, kidney, pancreas, mammary gland, skeletal muscle, salivary gland, and adipose tissue $(1,2)$. Moreover, such endogenous biotin sometimes interferes with immunohistochemical evaluation by the avidin-biotin-peroxidase complex (ABC) method, even in the case of formalin-fixed and paraffin-embedded tissue sections $(3,4)$.

In 1983, intranuclear inclusions were observed in endometrial glands during gestation and the puerperium by Mazur et al. (5), who called them "optically clear nuclei" on the basis of their histological 
and ultrastructural characteristics. Using biotinspecific antibodies and peroxidase-labeled avidin, Yokoyama et al. (6) and Sickel et al. (7) proved that such inclusions contain high level of biotin. Before the reports by Yokoyama et al. (6) and Sickel et al. (7), Tsujimoto et al. (8) described three cases of ovarian endometrioid adenocarcinoma with biotinrich intranuclear inclusions but without the morular structures known as morules in an elderly postmenopausal patient. Thereafter, such biotin-rich intranuclear inclusions were also found in the morules of well-differentiated fetal adenocarcinoma of the lung (WDFA), thyroid carcinoma, pancreatoblastoma, and colonic adenoma (9-13).

$\beta$-Catenin was identified initially as a protein associated with the cytoplasmic region of E-cadherin, which is a transmembrane protein that is involved in cell adhesion. Adhesive interactions between neighboring cells and with the extracellular matrix are major determinants of cell and tissue morphogenesis, and thus, it is likely that both $\beta$-catenin and E-cadherin play important roles in tissue and tumor morphology. $\beta$-Catenin is, in addition, a component of the Wnt signaling pathway, which is involved in tissue differentiation during embryonal development, and it also interacts with the product of the gene that is the genetic determinant of adenomatous polyposis coli (APC). Malfunction of the Wnt signaling pathway results in the intranuclear accumulation $\beta$-catenin and transcriptional activation of specific target genes, which include $c$-myc and cyclin D1, during embryonal development (14, 15). Based upon the observations that frequent mutations in the gene for APC and/or $\beta$-catenin were reported in morule-associated neoplasms (16-20) and that the morular area showed especially strong nuclear expression for $\beta$-catenin in WDFA (19), Nakatani and associates (19) recently proposed the idea that the intranuclear accumulation of $\beta$-catenin may be a common denominator for the development of morule-associated neoplasms.
In the present study, we performed an immunohistochemical analysis of neoplastic and nonneoplastic lesions with biotin-rich intranuclear inclusions to examine the correlation between such inclusions and the intracellular localization of biotin-binding enzymes and $\beta$-catenin.

\section{MATERIALS AND METHODS}

\section{Preparation of Tissues}

A variety of histopathologically diagnosed neoplastic and non-neoplastic lesions with biotin-rich intranuclear inclusions from various organs was examined in the present study (Table 1). The nonneoplastic samples consisted of 26 samples of pregnancy-related endometrium (e.g., endometrium at cesarean section, endometrium at abortion, and puerperal endometrium) and one ovarian endometriotic cyst from a patient who had delivered a baby 3 months before her oophorectomy. The neoplastic lesions consisted of five endometrioid adenocarcinomas of the uterus; two endometrioid adenocarcinomas of the ovary, including one case that had been described previously by one of the authors (MT) (8); two adenomas and two adenocarcinomas of the colon; two pyloric gland-type adenomas of the gallbladder; one pancreatoblastoma; one WDFA; and two samples of a cribriformmorular variant $(\mathrm{C}-\mathrm{M} / \mathrm{v})$ of thyroid carcinoma. In the present study, we considered the well-known "squamoid corpuscles" seen in pancreatoblastoma to be a type of morule. Additionally, conventional types carcinomas without morule, for instance, 10 uterine endometrioid adenocarcinomas, 10 colonic adenocarcinomas, 10 pulmonary adenocarcinomas, 5 papillary carcinomas of the thyroid, and 5 follicular carcinomas of the thyroid were used as controls.

All tissue samples were fixed in $10 \%$ formalin and embedded in paraffin by routine methods. Sections

TABLE 1. Results of Immunostaining for Biotin, Biotin-Binding Enzymes, and $\beta$-Catenin

\begin{tabular}{|c|c|c|c|c|c|c|c|c|}
\hline & $\begin{array}{l}\text { Number } \\
\text { of Cases }\end{array}$ & Morules & Biotin & PAC & PCC & $\mathrm{ACC}$ & CPS & $\beta$-Catenin \\
\hline \multicolumn{9}{|l|}{ Non-neoplastic lesions } \\
\hline Pregnancy-related endometrium & 26 & - & + & + & + & - & - & - \\
\hline Pregnancy-related ovarian endometriotic cyst & 1 & - & + & + & + & - & - & - \\
\hline \multicolumn{9}{|l|}{ Neoplastic lesions } \\
\hline Ovarian endometrioid adenocarcinoma & 2 & $-1+*$ & + & + & + & - & - & + \\
\hline Uterine endometrioid adenocarcinoma & 5 & + & + & + & + & - & - & + \\
\hline Colonic adenoma & 2 & + & + & + & + & - & - & + \\
\hline Colonic adenocarcinoma & 2 & + & + & + & + & - & - & + \\
\hline Pyloric gland-type adenoma of the gallbladder & 2 & + & + & + & + & - & - & + \\
\hline Pancreatoblastoma & 1 & + & + & + & + & - & - & + \\
\hline WDFA & 1 & + & + & + & + & - & - & + \\
\hline $\mathrm{C}-\mathrm{M} / \mathrm{v}$ of thyroid carcinoma & 2 & + & + & + & + & - & - & + \\
\hline
\end{tabular}

PAC, pyruvic acid carboxylase; PCC, propionyl CoA carboxylase; ACC, acetyl CoA carboxylase; CPS, carbamoyl phosphate synthetase I; WDFA, well-differentiated fetal adenocarcinoma of the lung; C-M/v, cribriform-morular variant.

* One case had morules while the other had none. 
were cut at 4- $\mu \mathrm{m}$ thickness and mounted on silanecoated glass slides. One section of each sample was stained with hematoxylin and eosin.

\section{Immunohistochemical Staining}

All paraffin sections were deparaffinized in xylene and rehydrated in a graded ethanol series. Endogenous peroxidase activity was eliminated by incubation with $3 \%$ hydrogen peroxide for $20 \mathrm{~min}$ utes at room temperature. The primary antibodies used in the study were a monoclonal biotin-specific antibody (1:100; DAKO, Carpinteria, CA, USA), a monoclonal $\beta$-catenin-specific antibody (1:100; Novocastra, Newcastle upon Tyne, UK), and individual rabbit polyclonal antisera against PAC, PCC, ACC and CPS, respectively. The latter four antisera had been generated at Oita Medical University by immunization of rabbits with chemically synthesized peptides that were based on amino acid sequences that had been reported previously. The sequences of peptides and the results of characterizations by immunoblotting and immunoelectron microscopy can be found in our previous reports $(21,22)$. Before immunostaining of $\beta$-catenin, PCC, and CPS, sections were heated in $10 \mathrm{~mm}$ citric acid buffer, $\mathrm{pH}$ 6.0, at $121^{\circ} \mathrm{C}$ for 15 minutes. After immersion in diluted normal goat serum (1:10) for 10 minutes, sections were incubated with primary antibodies or antisera for 2 hours at room temperature and rinsed with phosphate buffered saline. Then the sections were incubated for 30 minutes with second antibodies that had been conjugated with horseradish peroxidase-labeled polymer (Envision; DAKO). Reaction products were visualized with $0.02 \% 3,3$ '-diaminobenzidine tetrahydrochloride and $0.005 \%$ hydrogen peroxide. Nuclei were counterstained with Mayer's hematoxylin. Control sections, incubated with normal mouse IgG or preimmune rabbit serum instead of corresponding primary antibodies and antisera, gave negative results.

\section{Electron Microscopy}

For electron microscopy, we cut a small piece of fresh gestational endometrium into fragments and divided those into two groups for conventional electron microscopy and immunoelectron microscopy. Samples were processed for conventional electron microscopy by routine method. Samples for immunoelectron microscopy were dehydrated in an ascending graded series of ethanol, infiltrated with LR-White resin (London Resin Company Ltd, Berkshire, UK), and polymerized by heating at $55^{\circ} \mathrm{C}$ for 24 hours. Ultrathin sections of LR-White-embedded tissue were immersed in diluted normal goat serum (1:10) for 10 minutes to prevent the nonspecific binding of antibodies. Then, the biotinspecific monoclonal antibody (1:100) was applied to the sections, which were incubated for 2 hours at room temperature. Next, the sections were incubated with goat antibodies against mouse IgG that had been conjugated to 15-nm gold particles (1:10, Amersham, Little Chalfont, Buckinghamshire, UK) for 30 minutes at room temperature. Sections were finally counterstained with uranyl acetate and lead citrate in the standard manner.

\section{RESULTS}

The intranuclear inclusions in our series of samples were homogeneously pale, with a ground-glass appearance and occasional marginated chromatin, and looked like nuclei of herpesvirus-infected cells (Fig. 1-7, HE). The results of immunostaining are summarized in Table 1.

In all cases of pregnancy-related endometrium, we observed intranuclear inclusions in the endometrial glands, with the number of inclusions varying from case to case. The intranuclear inclusions were, without exception, immunohistochemically positive for biotin, PAC, and PCC, whereas they were negative for ACC, CPS, and $\beta$-catenin (Fig. 1). A case of ovarian endometriotic cyst gave identical results (Fig. 2).

All of the neoplastic lesions that we examined contained morular structures, with one exception. The single exception was an ovarian endometrioid adenocarcinoma from an elderly postmenopausal patient, and the specimen had numerous intranuclear inclusions in the glandular cells, despite the absence of morules. Most of the neoplastic glands were surrounded by numerous luteinized stromal cells with clear cytoplasm. The intranuclear inclusions in the glandular cells were positive for biotin, PAC, and PCC, whereas they were negative for ACC and CPS. Immunostaining for $\beta$-catenin was diffusely positive in the glandular nuclei, and the intensity of staining of inclusions was weaker than that of nuclei in other glandular cells (Fig. 3). In other neoplastic lesions, which included an ovarian endometrioid adenocarcinoma with morules, five uterine endometrioid adenocarcinomas, two colonic adenomas, two colonic adenocarcinomas, two pyloric gland-type adenomas of the gallbladder, one pancreatoblastoma, one WDFA, and two thyroid carcinomas of cribriform-morular variant, morular structures were clearly evident. The intranuclear inclusions were all positive for biotin, PAC, and PCC, whereas they were negative for ACC and CPS. Immunostaining for $\beta$-catenin was positive mainly in the constituent cells of morular structures, with obviously intense staining in the nuclei and faint staining in the cytoplasm (Figs. 4-7), and 

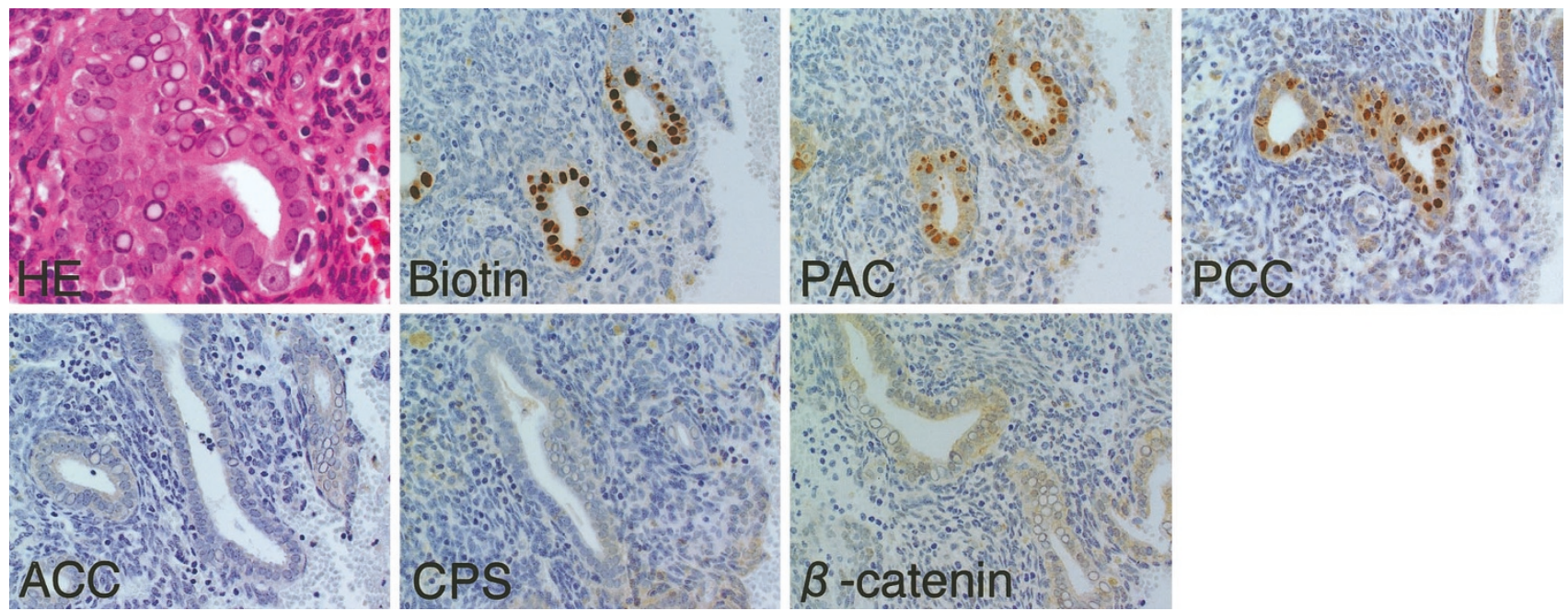

FIGURE 1. Pregnancy-related endometrium. Intranuclear inclusions are immunopositive for biotin, pyruvic acid carboxylase (PAC), and propionyl CoA carboxylase (PCC) but immunonegative for acetyl CoA carboxylase (ACC), carbamoyl phosphate synthetase I (CPS), and $\beta$-catenin.
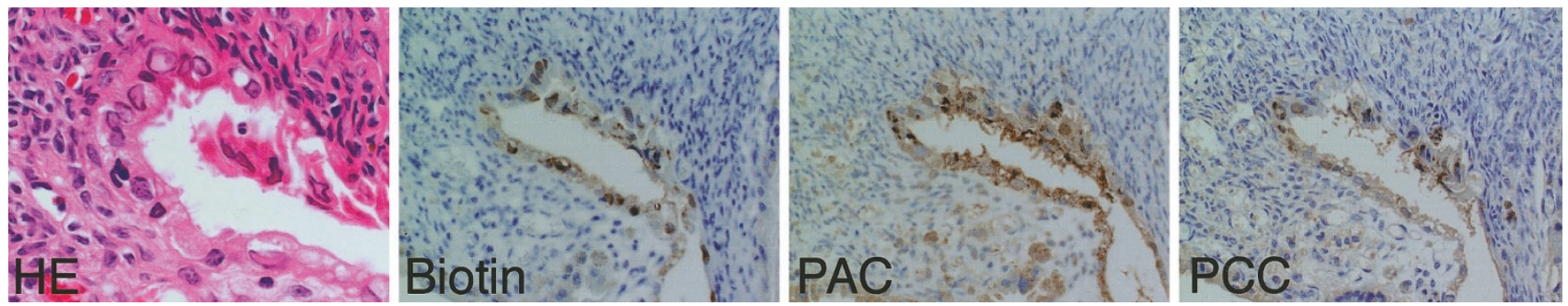

FIGURE 2. Ovarian endometriotic cyst. Glandular cells have intranuclear inclusions that are immunopositive for biotin, PAC, and PCC.
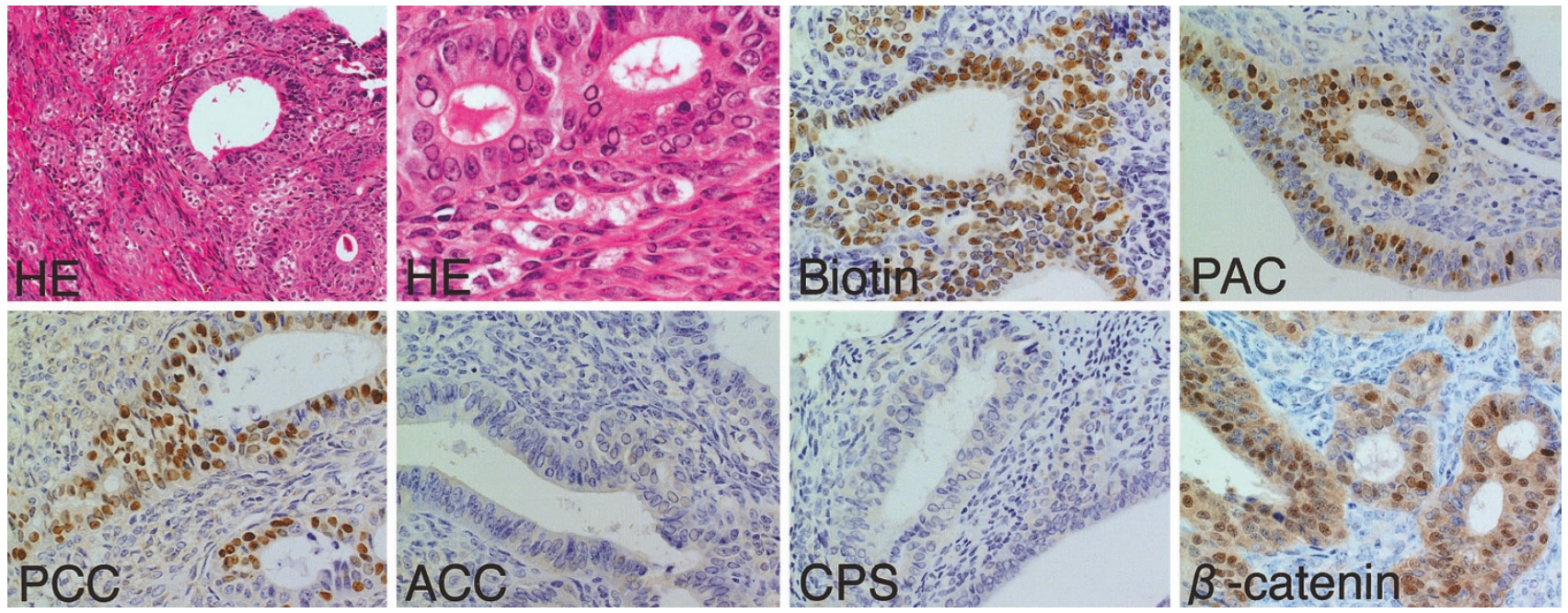

FIGURE 3. Ovarian endometrioid adenocarcinoma without morules. Neoplastic glands are surrounded by luteinized cells. Intranuclear inclusions in the neoplastic cells are immunopositive for biotin, PAC, PCC, and $\beta$-catenin but immunonegative for ACC and CPS.

there was occasional staining in glands outside morules. Intranuclear expression of $\beta$-catenin mentioned above was found in both the neoplastic cells with and without the intranuclear inclusion.

Of control neoplastic samples without morules, intranuclear expression of $\beta$-catenin was focally seen in eight colonic adenocarcinoma and one case of uterine endometrioid adenocarcinoma but not in other samples. There was no biotin-rich intranu- clear inclusion in these conventional types of carcinoma without morules.

In terms of ultrastructure, the intranuclear inclusions were composed entirely of microfilaments that were distributed in a fascicular or fingerprintlike fashion (Fig. 8A). Furthermore, numerous gold particles, evidence of the presence of immunoreactive biotin, were observed in the filamentous areas (Fig. 8B). 


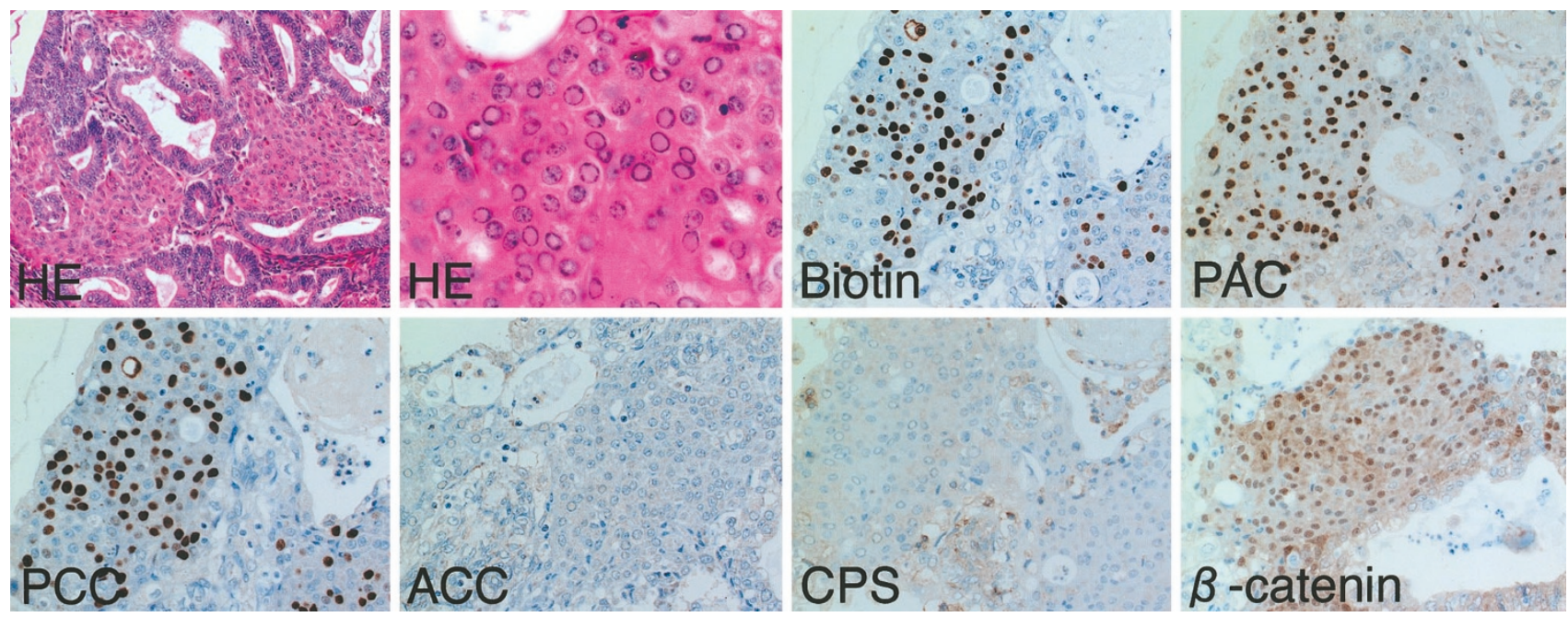

FIGURE 4. Uterine endometrioid adenocarcinoma. Numerous intranuclear inclusions are visible in the morules, which are immunopositive for biotin, PAC, PCC, and $\beta$-catenin but immunonegative for ACC and CPS.
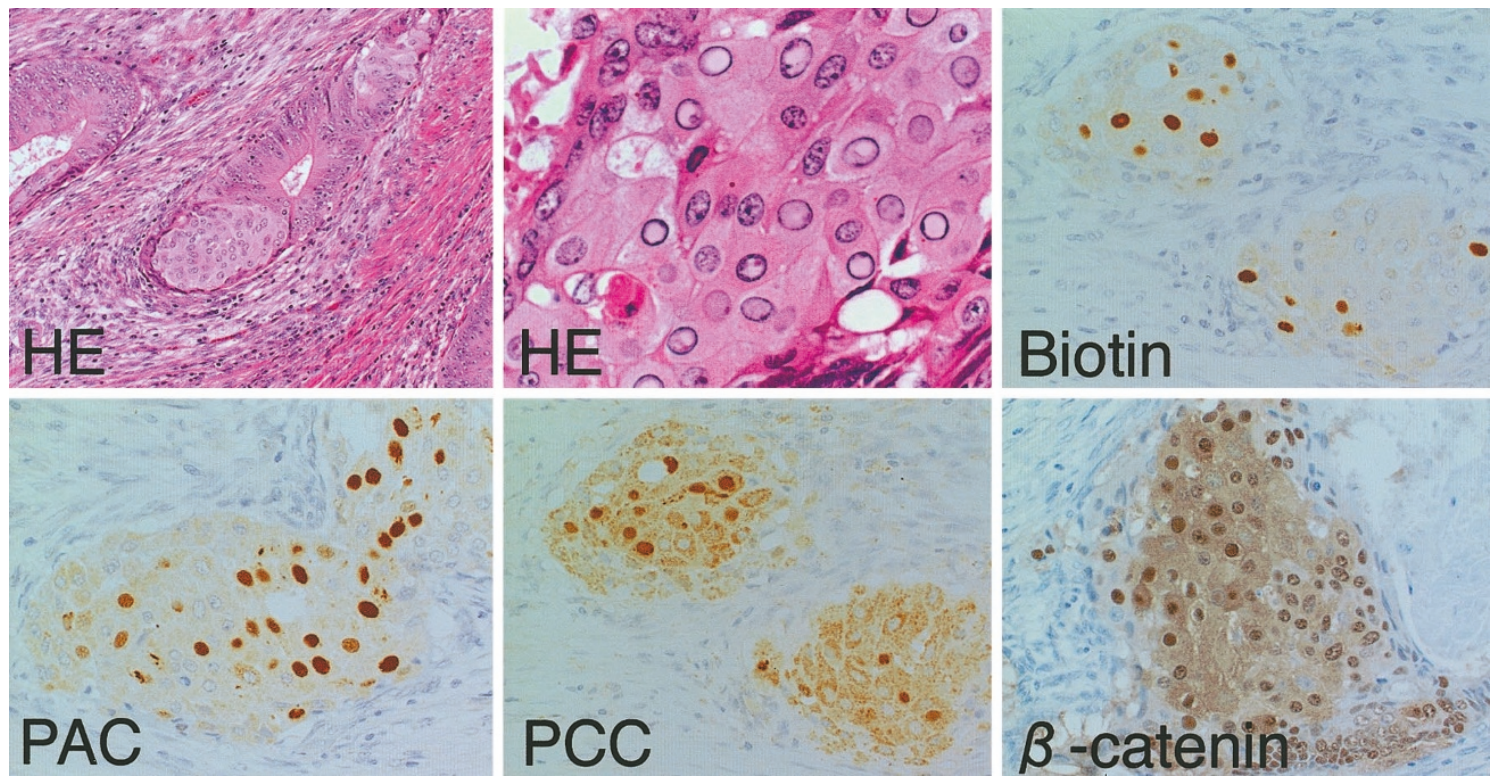

FIGURE 5. Colonic adenocarcinoma. The morules in the neoplastic glands are immunopositive for biotin, PAC, PCC, and $\beta$-catenin.

\section{DISCUSSION}

Biotin-rich intranuclear inclusions have been observed in a variety of neoplastic lesions and pregnancy-related endometrium. It is important to know this finding, especially in evaluating intranuclear antigens, because they look like nuclei of herpesvirus-infected cells and cause a false-positive immunohistochemical result by the ABC method. All lesions with such inclusions that have been reported to date were included in this study, as well as some that have not previously been reported. Morphologically similar intranuclear inclusions have occasionally been found in neoplastic and non-neoplastic lesions such as papillary carcinoma of the thyroid, bronchioloalveolar carcinoma, and hepatocellular carcinoma, as well as in gastroduo- denitis and cholecystitis. In our experience, however, these intranuclear inclusions do not contain biotin.

Biotin is a coenzyme that is essential for reactions catalyzed by carboxylases, transcarboxylases, and decarboxylases. Of the four enzymes examined in this study, PAC and PCC were found in all biotinrich intranuclear inclusions that we identified. Tsujimoto et al. (8) found two biotin-binding molecules, with molecular masses of 69 and $72 \mathrm{kDa}$, respectively, in the nuclear fraction of ovarian endometrioid carcinoma with biotin-rich intranuclear inclusions. These macromolecules are almost identical in terms of molecular mass to the $\alpha$ subunits of MCC and PCC. Furthermore, Fleming et al. (23) induced the formation of biotin-rich multinucle- 


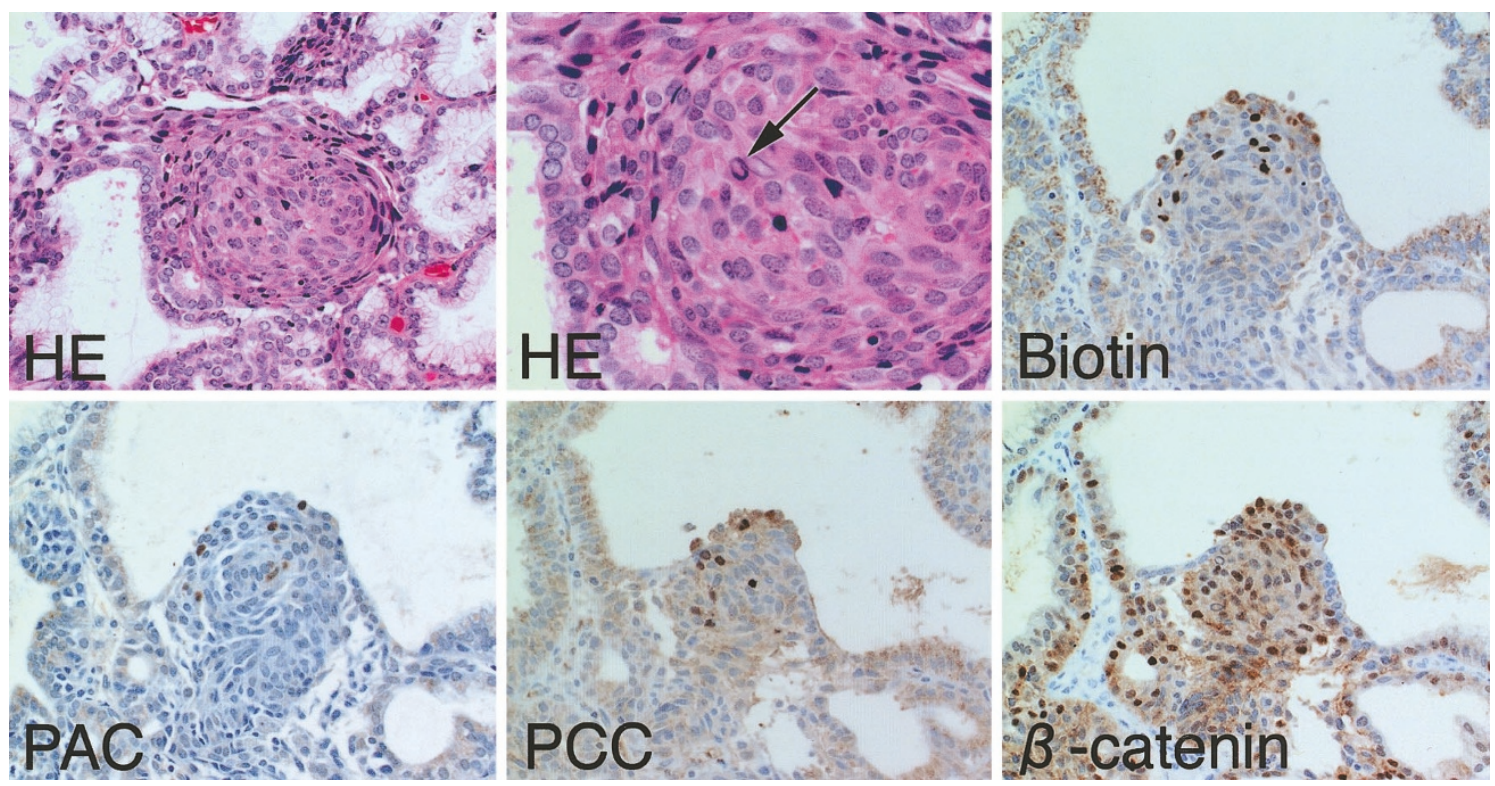

FIGURE 6. Pyloric gland-type adenoma of the gallbladder. Round morules with scattered intranuclear inclusions (arrow) in the tumor are immunopositive for biotin, PAC, and PCC. Immunostaining for $\beta$-catenin is positive in the nuclei of the morules, as well as in those of surrounding neoplastic cells.
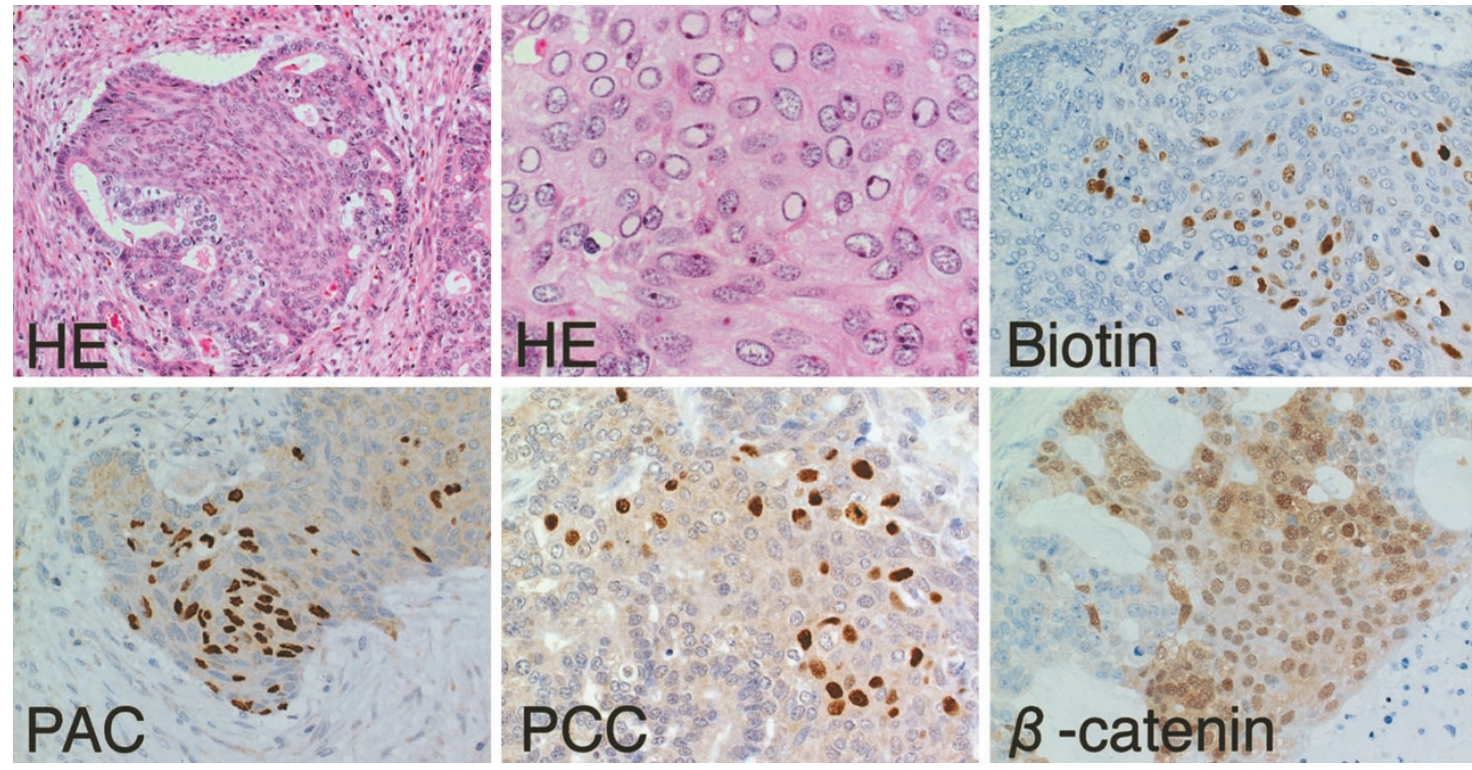

FIGURE 7. Pancreatoblastoma. A morular structure, generally referred to as a squamoid corpuscle, has intranuclear inclusions that are immunopositive for biotin, PAC, PCC, and $\beta$-catenin.

ated structures in differentiating human endometrial cells cultured with fresh fetal bovine serum, and these structures contained PAC, PCC, and MCC. Our demonstration, by immunohistochemical staining, that biotin-rich intranuclear inclusions contained substantial amounts of PAC and PCC is consistent with these earlier reports. However, both enzymes are known to catalyze reactions in gluconeogenesis and to be localized in mitochondria, and it is unclear why and how mitochondrial biotin-binding enzymes that are involved in gluconeogenesis might migrate from the cytoplasm to the nucleus.
In a previous study, we showed that biotin-rich intranuclear inclusions in endometrial glands during gestation and the puerperium become evident during the 16th week of gestation and can be detected until 37 days postpartum (6). The ovarian endometriotic cyst in our series appears to have been related to pregnancy because the patient had delivered 3 months before her oophorectomy. A similar case of ovarian endometriosis during pregnancy was reported by Sakaki et al. (24). In addition, the endometrioid adenocarcinoma without morules that is shown in Figure 3 was associated with numerous luteinized cells that surrounded the 


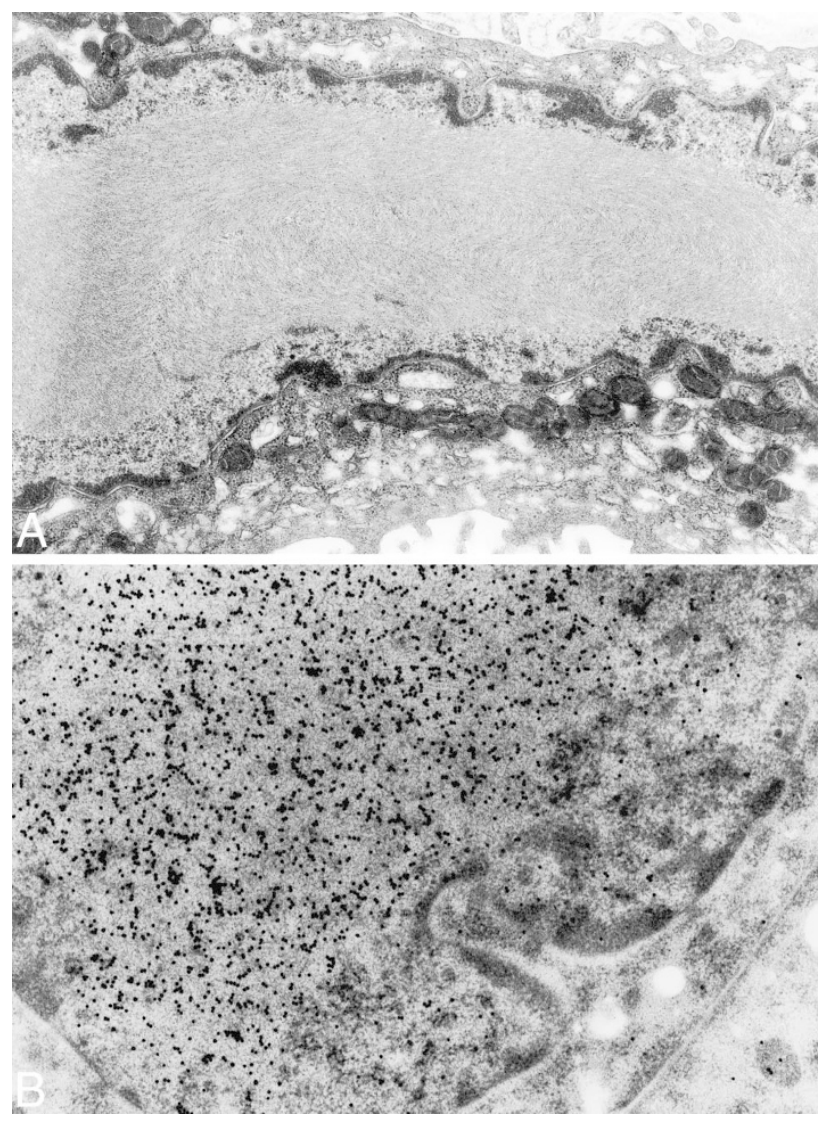

FIGURE 8. An intranuclear inclusion from a case of gestational endometrium is completely occupied by microfilaments (A; original magnification, $9400 \times$ ). Numerous gold particles indicative of the presence of biotin are visible in the filamentous area $(\mathbf{B}$; original magnification, $13,000 \times$ ).

neoplastic glands. Given that such luteinized cells secrete progesterone, we can infer that the ovarian tumor was hormonally under conditions analogous to pregnancy. The report by Roberts et al. (25) on the induction of endometrial intranuclear inclusions by progesterone supports this inference.

In our series, we detected aberrant intranuclear expression of $\beta$-catenin in all neoplastic lesions with intranuclear inclusions. Furthermore, all of the lesions were associated with formation of morules with the exception of one case of endometrioid adenocarcinoma without morules, which seemed to have developed under conditions analogous to pregnancy, as mentioned above. In other words, all pregnancy-unrelated neoplastic lesions were associated with morules. Conversely, attention should be paid to intranuclear inclusions that include biotin and $\beta$-catenin when morules are observed in a tumor. Nonetheless, morules do not necessarily contain the intranuclear inclusions. We have, in fact, come across several cases in which no intranuclear inclusions were seen, even though the tumors were associated with morules. However, no such cases were included in the present study.
Mutations in genes for $\beta$-catenin and APC have been detected at high frequency in the neoplastic lesions included in our series, such as endometrioid adenocarcinoma of the uterus and ovary, C-M/v of thyroid carcinoma, colonic adenoma/adenocarcinoma, pyloric gland-type adenoma of the gallbladder, WDFA, and pancreatoblastoma (16-20, 26-29). The Wnt signaling pathway, mediated by $\beta$-catenin, is involved in tissue differentiation as well as carcinogenesis $(14,15)$. It is possible that $\beta$-catenin participates in the morular formation as reported by Nakatani et al. (19), although the role of $\beta$-catenin in these neoplastic lesions remains unclear. We analyzed the mutation in $\beta$-catenin in one case of ovarian endometrioid adenocarcinoma without morules and in two cases of colonic adenocarcinoma, of which we had sufficient tissue for amplification of DNA by the polymerase chain reaction. However, the template DNA was decayed probably during the fixation and/or storage and the amplification was not informative in any of these cases (data not shown).

In conclusion, lesions with biotin-rich intranuclear inclusions could be divided into two categories. Non-neoplastic/pregnancy-related endometrial lesions were associated neither with morules nor with the intranuclear accumulation of $\beta$-catenin. By contrast, neoplastic lesions were unrelated to pregnancy but were associated with morules and the intranuclear accumulation of $\beta$-catenin. In both categories, the intranuclear inclusions contained mitochondrial biotin-binding enzymes (PAC and PCC) and biotin. In the neoplastic/pregnancy-unrelated category, furthermore, most of the neoplastic lesions seem to be frequently correlated with mutations in genes for APC and $\beta$-catenin, according to the previous reports $(16-20,26-29)$. The single exceptional case was an ovarian endometrioid adenocarcinoma with numerous luteinized stromal cells, in which the intranuclear accumulation of $\beta$-catenin was observed despite the absence of morules. In terms of pathogenesis, this tumor can, perhaps, be placed in the non-neoplastic/pregnancy-related endometrial category rather than the neoplastic/pregnancyunrelated category, because adjacent luteinized cells might well have secreted progesterone. The neoplastic/pregnancy-unrelated lesions can also be called the morular category, aside from this single exceptional case. There may be a hormonal response participating in the formation of biotin-rich intranuclear inclusions even in morular structures.

This is the first report, to our knowledge, of the immunohistochemical demonstration of the intranuclear co-expression of biotin and biotinbinding enzymes (PAC and PCC) in a variety of neoplastic and non-neoplastic lesions with intranuclear inclusions. Although the role of $\beta$-catenin in 
morule-associated neoplastic lesions, the relationship between $\beta$-catenin and biotin/biotin-binding enzymes, and the mechanism of migration of biotin and biotin-binding enzymes from the cytoplasm to the nucleus remain unclear, the present study provides a basis for further clarification of the mechanism of formation of biotin-rich intranuclear inclusions and morules.

Acknowledgments: The authors thank Dr. N. Kaku, Mr. S. Yano, and Ms. T. Nakamatsu for their skilled technical assistance; Dr. S. Takeno and Dr. T. Sato for their valuable comments on the manuscript; and Dr. K. Tsuji (Oita Prefectural Hospital, Oita, Japan), Dr. Y. Takana (Kanagawa Children's Medical Center, Kanagawa, Japan), and Dr. T. Ito (Yokohama City University, Yokohama, Japan) for their assistance in providing pathologic materials.

\section{REFERENCES}

1. Dakshinamurti K, Mistry SP. Tissue and intracellular distribution of biotin-COOH in rats and chicks. J Biol Chem 1963;238:294-6.

2. Wood GS, Warnke R. Suppression of endogenous avidinbinding activity in tissues and its relevance to biotin-avidin detection systems. J Histochem Cytochem 1981;29:1196-204.

3. Bussolati G, Gugliotta P, Volante M, Pace M, Papotti M. Retrieved endogenous biotin: a novel marker and a potential pitfall in diagnostic immunohistochemistry. Histopathology 1997;31:400-7.

4. Kashima K, Yokoyama S, Daa T, Nakayama I, Nickerson PA, Noguchi S. Cytoplasmic biotin-like activity interferes with immunohistochemical analysis of thyroid lesions: a comparison of antigen retrieval methods. Mod Pathol 1997;10:515-9.

5. Mazur MT, Hendrickson MR, Kempson RL. Optically clear nuclei: an alteration of endometrial endometrium in the presence of trophoblast. Am J Surg Pathol 1983;7:415-23.

6. Yokoyama S, Kashima K, Inoue S, Daa T, Nakayama I, Moriuchi A. Biotin-containing intranuclear inclusions in endometrial glands during gestation and puerperium. Am J Clin Pathol 1993;99:13-7.

7. Sickel JZ, di Sant'Agenese PA. Anomalous immunostaining of "optically clear" nuclei in gestational endometrium; a potential pitfall in the diagnosis of pregnancy-related herpesvirus infection. Arch Pathol Lab Med 1994;118:831-3.

8. Tsujimoto M, Noguchi M, Taki I. Immunohistochemical and electromicroscopic study of the intranuclear inclusion bodies containing biotin in the ovarian endometrioid carcinoma. J Clin Electron Microsc 1991;24:5-6.

9. Cameselle-Teijeiro J, Chan JKC. Cribriform-morular variant of papillary carcinoma: a distinctive variant representing the sporadic counterpart of familial adenomatous polyposisassociated thyroid carcinoma? Mod Pathol 1999;12:400-11.

10. Nakatani Y, Kitamura H, Inayama Y, Ogawa N. Pulmonary endodermal tumor resembling fetal lung: the optically clear nucleus is rich in biotin. Am J Surg Pathol 1994;18:637-42.

11. Okamoto Y, Kashima K, Daa T, Yokoyama S, Nakayama I, Noguchi S. Morule with biotin-containing intranuclear inclusions in thyroid carcinoma. Pathol Int 1995;45:573-9.

12. Sasaki A, Yokoyama S, Arita T, Inomata M, Kashima K, Nakayama I. Morules with biotin-containing optically clear nuclei in colonic tubular adenoma. Am J Surg Pathol 1999;23: 336-41.
13. Tanaka Y, Ijiri R, Yamanaka S, Kato K, Nishihira H, Nishi T, et al. Pancreatoblastoma: optically clear nuclei in squamoid corpuscles are rich in biotin. Mod Pathol 1998;11:945-9.

14. Ben-Ze'ev A, Shtutman M, Zhurinsky J. The integration of cell adhesion with gene expression: the role of $\beta$-catenin. Exp Cell Res 2000;261:75-82.

15. Novak A, Dedhar S. Signaling through $\beta$-catenin and Lef/Tcf. Cell Mol Life Sci 1999;56:523-37.

16. Abraham SC, Wu TT, Klimstra DS, Finn LS, Lee JH, Yeo CJ, et $a l$. Distinctive molecular genetic alteration in sporadic and familial adenomatous polyposis-associated pancreatoblastomas. Frequent alteration in the APC/ $\beta$-catenin pathway and chromosome 11p. Am J Pathol 2001;159:1619-27.

17. Cameselle-Teijeiro J, Ruiz-Ponte C, Loidi L, SuarezPenaranda J, Baltar J, Sobrinho-Simoes M. Somatic but not germline mutation of the APC gene in a case of cribriformmorular variant of papillary thyroid carcinoma. Am J Clin Pathol 2001;115:486-93.

18. Gamallo C, Palacios J, Moreno G, Calvo de Mora J, Suarez A, Armas A. $\beta$-Catenin expression pattern in stage I and II ovarian carcinomas. Relationship with $\beta$-catenin gene mutations, clinicopathological features, and clinical outcome. Am J Pathol 1999;155:527-36.

19. Nakatani Y, Masudo K, Miyagi Y, Inayama Y, Kawano N, Tanaka Y, et al. Aberrant nuclear localization and gene mutation of $\beta$-catenin in low-grade adenocarcinoma of fetal lung type: up-regulation of the Wnt signaling pathway may be a common dominator for the development of tumors that form morules. Mod Pathol 2002;15:617-24.

20. Saegusa M, Okayasu I. Frequent nuclear $\beta$-catenin accumulation and associated mutations in endometrioid-type endometrial and ovarian carcinomas with squamous differentiation. J Pathol 2001;194:59-67.

21. Lu CS, Kashima K, Daa T, Yokoyama S, Yanagisawa S, Nakayama I. Immunohistochemical study of the distribution of endogenous biotin and biotin-binding enzymes in ductal structures of salivary gland tumours. J Oral Pathol Med 2000; 29:445-51.

22. Sato T, Kashima K, Gamachi A, Daa T, Nakayama I, Yokoyama S. Immunohistochemical localization of pyruvate carboxylase and carbamyl-phosphate synthetase I in normal and neoplastic human pancreatic tissues. Pancreas 2002;25: $130-5$.

23. Fleming H, Condon R, Peterson G, Guck I, Prescott E, Chatfield $\mathrm{K}$, et al. Role of biotin-containing membranes and nuclear distribution in differentiating human endometrial cells. J Cell Biochem 1998;71:400-15.

24. Sakaki M, Hirokawa M, Tezuka K. Ovarian endometriosis showing decidual change and Arias-Stella reaction with biotin-containing intranuclear inclusions. Acta Cytol 2003; 47:321-4.

25. Roberts DK, Horbelt DV, Powell LC. The ultrastructural response of human endometrium to medroxyprogesterone acetate. Am J Obstet Gynecol 1975;15:811-8.

26. Munemitsu S, Albert I, Souza B, Rubinfeld B, Polakis P. Regulation of intracellular $\beta$-catenin levels by the adenomatous polyposis coli (APC) tumor-suppressor protein. Proc Natl Acad Sci U S A 1995;92:3046-50.

27. Samowitz WS, Powers MD, Spirio LN, Nollet F, van Roy F, Slattery ML. Beta-catenin mutations are more frequent in small colorectal adenomas than in larger adenomas and invasive carcinomas. Cancer Res 1999;59:1442-4.

28. Sparks AB, Morin PJ, Vogelstein B, Kinzler KW. Mutational analysis of the APC/ $\beta$-catenin/Tcf pathway in colorectal cancer. Cancer Res 1998;58:1130-4.

29. Yanagisawa N, Mikami T, Saegusa M, Okayasu I. More frequent $\beta$-catenin exon 3 mutations in gallbladder adenomas than in carcinomas indicate different lineages. Cancer Res 2001;61:19-22. 